\title{
Spin-Orbit Splitting in the $N-4$ Spectra and the Deformed Baryon Model
}

\author{
M.V.N. Murthy, M. Brack ${ }^{1}$, R.K. Bhaduri, B.K. Jennings ${ }^{2}$ \\ Physics Department, McMaster University, Hamilton, Ontario, Canada L8S 4M1
}

Received 4 February 1985; in revised form 12 June 1985

\begin{abstract}
The absence of appreciable spin-orbit splitting in the low-lying even and odd parity states of the nucleon and delta is puzzling in conventional quark models. A constitutent quark model, in which the quarks interact through gluon as well as pion exchange, and the baryon is allowed to deform in the excited states, may provide a solution to the problem.
\end{abstract}

\section{Introduction}

There is a wealth of experimental data [1] in baryon spectroscopy that should constitute a good testing ground for nonperturbatice QCD. At the present time, however, it is not possible to confront the data from a fundamental theoretical point of view. Rather, a number of quark models have been developed [2-6] with some ingredients of the theory put in phenomenologically. These are either nonrelativistic constituent quark models $[2,3]$ with a suitably chosen interaction potential, or relativistic bag-like models [4-6] where the interaction is treated perturbatively. There are also variants of the constituent quark model with relativistic kinematics included in a variational calculation for the excited states [7]. A comprehensive analysis of the data has been made with constituent quark models $[2,3,7$, 8] and to a lesser degree, with M.I.T. [4] and chiral $[5,6]$ bag models. Recently, the nonrelativistic three-body Faddeev equations have also been solved [9] for the excited states of baryons with a phenomenological two-body interaction. In a consistent

1 Permanent address: Theoretical Physics, University of Regensburg, D-8400 Regensburg, FRG

2 Permanent address: TRIUMF, 4004 Wesbrook Mall, Vancouver, Canada V6T $2 \mathrm{~A} 3$ model, both the low-lying odd- and even-parity states should be reproduced simultaneously, and moreover the near-absence of the spin-orbit splitting in the observed spectra should be explained. In this paper, this is attempted for the $N$ and $\Delta$ states using a model in which the baryon is allowed to deform in the excited states $[10,11]$, and the quarks interact via gluons as well as pions. The rationale of such a model is discussed in [12].

In this paper, we would concentrate on the spinorbit problem. Experimentally, the $N-A$ spectrum shows practically no spin-orbit splitting, except between the $\Delta(1,620) \frac{1}{2}^{-}$and $\Delta(1,700) \frac{3}{2}^{-}$states. This is a serious problem both in the bag model and the constituent quark model. We shall concentrate on the nonrelativistic models. In spectroscopic calculations, the $q-q$ interaction that is diagonalised is assumed to arise from the one-gluon exchange mechanism. This force has spin-spin, tensor and spin-orbit components whose strengths are determined by the quark-gluon coupling constant, $\alpha_{s}$. With an oscillator spacing of $\hbar \omega \approx 500 \mathrm{MeV}$ and the constituent quark masses, one has to take $\alpha_{s} \approx 1$ to reproduce the observed $N-\Delta$ ground state splitting. This value of $\alpha_{s}$ is about three times larger than the estimate of the QCD running coupling constant at $q^{2}=1 \mathrm{GeV}^{2}$. It has also the very undesirable effect of introducing large spin-orbit splittings. For example, although the odd-parity $(N=1) \Delta$ states $\left(\frac{1}{2}^{-}\right.$and $\left.\frac{3}{2}^{-}\right)$ are split by about the right amount, the nucleon $l$ $=1, S=\frac{3}{2}$ states with differing $J$ 's become separated by more than $500 \mathrm{MeV}$. Isgur and Karl [2] pointed out that there may be delicate cancellation between the one-gluon-exchange spin-orbit force and the spin-orbit interaction arising from the Thomas term of the scalar confining potential. This is true, but it does not solve the problem. An appropriate strength of the Thomas term may indeed cancel the spin- 
orbit splitting from the one-gluon exchange in the odd parity nucleon states, but it also enhances the splitting between the odd parity $\Delta$ states to an unacceptable degree. Moreover, it is unlikely that such delicate cancellations should persist in the $N=2$ even-parity states. Isgur and Karl [2] recognised these difficulties and decided to drop the spin-orbit interaction altogether. The spin-orbit puzzle in the odd-parity states has been recently carefully studied by Gromes [13]. He suggested that spin-orbit forces may also arise from the nonlocality of the confining scalar potential. An additional parameter characterising this nonlocality may consistently explain the puzzle for the $N=1$ states. However, more parameters are necessary to specify the nonlocality for the even-parity $N=2$ states, which were not examined by Gromes.

In this paper, we assume that the quarks couple not only to the gluons, but also to pions. This point of view has been advocated both in the bag model context, and in relativistic potential models by various authors [14-16]. It has also been examined in the nonrelativistic constituent quark-model approach [17-19] for the ground state properties of baryons, and in the derivation of the $N-N$ potential from $q-q$ interaction [20]. So far as spectroscopy is concerned, the effect of pion-coupling has only been examined for the low lying odd-parity states of the $N$ and $\Delta$ in the chiral bag model $[5,6]$, but no one has examined both the odd- and even-parity states in a single framework. In this paper we do so, with particular emphasis on the spin-orbit problem. The constituent quarks interact not only via the onegluon-exchange potential (OGEP), but also through one-pion exchange (OPEP). An appropriate pion form-factor is chosen to take account of the pion size, although this is not crucial as will be explained later. The contributions of the self-energy effects, due to the emission and absorption of a pion (or a gluon) by the same quark, are neglected: these are all lumped together in a constant constituent mass of the quark. This is not justifiable, as self-energy effects are state-dependent, and should therefore have a bearing on the spectroscopy of the states. Such calculations, however, have inherent difficulties [21], and we choose to retain only the lowest order interquark potential in our model. With this assumption, somewhat more than half the $N-\Delta$ ground state splitting arises from OPEP, which has no spin-orbit component. We then require the quark-gluon coupling $\alpha_{s} \approx 0.35$, consistent with the QCD estimate, to reproduce the observed $N-\Delta$ mass difference. This cuts down the one-gluon-exchange spin-orbit strength by about a factor of three. The scalar one-body confinement also gives rise to a spin-orbit splitting, whose magnitude is estimated from bag model calculations for the oddand even-parity states. When deformed orbitals are used, we find a further suppression in the spin-orbit matrix-elements, specially for the even parity excited states which are more deformed. We shall demonstrate in this paper that these two ingredients deformation and pion coupling to quarks, may consistently reproduce the observed $N-\Delta$ spectrum.

It is worth mentioning that the above Hamiltonian with gluon and pion exchange would not fit even the ground states of strange baryons. A fair fit to both the ground and excited states of strange baryons is possible only if kaon-exchange is also introduced between a strange and a nonstrange quark. Such a calculation is in progress.

The deformed mean-field model is described briefly in Sect. 2. In Sect. 3, the residual interactions in the Hamiltonian are given, and the method of calculation delineated. In the final section, the results are presented and compared with the data. The Appendix lists the relevant expressions for the matrix-elements of the interaction.

\section{The Deformed Model}

The basic features of the model first proposed by Bhaduri et al. [10] have been discussed in detail in [11]. For the sake of completeness we will discuss the relevant aspects here. In this model the valence quarks are assumed to be moving in a deformable mean field. The deformed eigenfunctions of this mean field provide the basis for diagonalising the residual two-quark interaction due to the one gluon exchange (OGE) and the one pion exchange (OPE) including the non-central components.

The mean field is state dependent and therefore some of the states could be non-orthogonal. The diagonalisation of the residual interaction in this nonorthogonal basis restores orthogonality through configuration mixing. The resulting eigenvalues and eigenstates in our model represent the masses and the structure of resonances in the baryon spectrum. The mean field Hamiltonian of the three-quark system, after eliminating the centre of mass terms, can then be written as [11]

$H_{0}=\frac{1}{2 m}\left(p_{\rho}^{2}+p_{\lambda}^{2}\right)+\frac{m}{2} \sum_{i=x, y, z} \omega_{i}^{2}\left(\rho_{i}^{2}+\lambda_{i}^{2}\right)$

where $m=m_{u}=m_{d}$, and the relative coordinates are

$\boldsymbol{\rho}=\frac{1}{\sqrt{2}}\left(\mathbf{r}_{1}-\mathbf{r}_{2}\right) ; \quad \lambda=\frac{1}{\sqrt{6}}\left(\mathbf{r}_{1}+\mathbf{r}_{2}-2 \mathbf{r}_{3}\right)$. 
Table 1. The intrinsic states in terms of the deformed oscillator excitation quanta $N=N_{x}+N_{y}+N_{z}$. A given set of $N_{x}, N_{y}, N_{z}$ defines an intrinsic state, the first few of which are listed in the first column. The $S U$ (6) multiplet structure to which it can couple is shown in the second column. The equilibrium shape is shown in the third column. The corresponding eigenenergies of $H_{0}$ at minimum, the moment of inertia $\mathscr{I}$, and $\left\langle L^{2}\right\rangle$ are shown in the next three columns respectively

\begin{tabular}{|c|c|c|c|c|c|}
\hline $\begin{array}{l}\text { Number of } \\
\text { excitation, } N\end{array}$ & $\begin{array}{l}\text { Multiplet } \\
\text { structure } \\
\text { (parity) }\end{array}$ & $\begin{array}{l}\text { Equilibrium } \\
\text { configuration }\end{array}$ & $\begin{array}{l}\text { Intrinsic } \\
\text { energy }\end{array}$ & $\frac{\hbar^{2}}{2 \mathscr{I}}$ & $\left\langle L^{2}\right\rangle$ \\
\hline $\begin{array}{l}N=0 \\
N_{x}=N_{y}=N_{z}=0\end{array}$ & {$\left[56^{+}\right]$} & $\begin{array}{l}\omega_{x}=\omega_{y}=\omega_{z} \\
\text { (spherical) }\end{array}$ & $3 h \omega_{0}$ & - & 0 \\
\hline $\begin{array}{l}N=1 \\
N_{x}=N_{y}=0, N_{z}=1\end{array}$ & {$\left[70^{-}\right]$} & $\begin{array}{l}\omega_{x}=\omega_{y}=2 \omega_{z} \\
\text { (prolate) }\end{array}$ & $3.780 h \omega_{0}$ & $0.126 h \omega_{0}$ & 3 \\
\hline $\begin{array}{l}N=2 \\
N_{x}=N_{y}=0, N_{z}=2\end{array}$ & {$\left[56^{+}\right],\left[70^{+}\right]$} & $\begin{array}{l}\omega_{x}=\omega_{y}=3 \omega_{z} \\
\text { (prolate) }\end{array}$ & $4.327 h \omega_{0}$ & $0.072 \hbar \omega_{0}$ & 8 \\
\hline$N_{x}=N_{y}=1, N_{z}=0$ & {$\left[20^{+}\right]$} & $\begin{array}{l}\omega_{x}=\omega_{y} \\
\text { (oblate) }\end{array}=\frac{\omega_{z}}{2}$ & $4.762 h \omega_{0}$ & $0.159 h \omega_{0}$ & 3 \\
\hline $\begin{array}{l}N=3 \\
N_{x}=N_{y}=0, N_{z}=3\end{array}$ & {$\left[56^{-}\right],\left[70^{-}\right]$} & $\begin{array}{l}\omega_{x}=\omega_{y}=4 \omega_{z} \\
\text { (prolate) }\end{array}$ & $4.762 h \omega_{0}$ & $0.047 \hbar \omega_{0}$ & 15 \\
\hline
\end{tabular}

The momenta conjugate to $\rho$ and $\lambda$ are $\mathbf{p}_{\rho}$ and $\mathbf{p}_{\lambda}$ respectively. Minimisation of the energy with the constraint

$\omega_{x} \omega_{y} \omega_{z}=\omega_{0}^{3}$

yields the condition [22]

$\omega_{x}\left(N_{x}+1\right)=\omega_{y}\left(N_{y}+1\right)=\omega_{z}\left(N_{z}+1\right)$

Here $N_{x}$ is the total number of excitation quanta in the $x$-direction, i.e. $N_{x}=N_{\rho_{x}}+N_{\lambda_{z}}$, and similarly for $N_{y}$ and $N_{z}$.

The intrinsic energy $E_{0}$ at equilibrium configuration is reproduced in Table 1 for $N=0,1,2$ and the lowest $N=3$ excitations of the quark orbitals. Notice that both for $N=1$ and 2 only the axially symmetric solutions can yield equilibrium configurations while $N=0$ remains spherically symmetric. In the case of $N=3$ the lowest possible configuration remains axially symmetric while the triaxial and symmetric solutions occur at higher energies. Throughout this analysis the axis of symmetry is chosen to be the $z$-axis.

We now proceed to construct the eigenstates of the Hamiltonian (2.1). To this end we define

$\psi_{N_{\rho_{x}} N_{\rho_{y}} N_{\rho_{z}}}(\rho)=\phi_{N_{\rho_{x}}}\left(\omega_{x}, \rho_{x}\right) \phi_{N_{\rho_{y}}}\left(\omega_{y}, \rho_{y}\right) \phi_{N_{\rho_{z}}}\left(\omega_{z}, \rho_{z}\right)$,

where $\phi_{N_{\rho_{i}}}\left(\omega_{i}, \rho_{i}\right)$ is the one dimensional oscillator eigenfunction, and similarly in $\lambda$. Denoting the total number of excitations in deformed orbitals as $N$, we have

$N=\sum_{i}\left(N_{\rho_{i}}+N_{\lambda_{i}}\right)$
When $N=0$, for example, the spatial wave function is,

$\Psi_{\mathrm{sym}}^{N=0}(\boldsymbol{\rho}, \lambda)=\psi_{000}(\boldsymbol{\rho}) \psi_{000}(\lambda)$.

The excited state wavefunctions for $N=1$ and 2 are given explicitly in [11] and will be used in this paper.

To evaluate matrix-elements between states of good $L$ and $J$, it is convenient to expand the deformed oscillator states in the basis of a spherical oscillator of frequency $\omega_{0}$, as defined in (2.3). For example, the deformed oscillator state in the $\rho$-coordinate, with $N_{\rho_{x}}=N_{\rho_{y}}=0$ and $N_{\rho_{z}}=\tilde{N}$, may be written as

$\psi_{00 \tilde{N}}(\rho)=\sum_{n_{\rho} l_{\rho}} C_{n_{\rho} l_{\rho}}^{(\tilde{N})} \phi_{n_{\rho} l_{\rho} m_{\rho}=0}(\rho)$,

where $\phi_{n l m}(\rho)$ are the usual spherical oscillator eigenstates and $C_{n l}^{(\tilde{N})}$ are the expansion coefficients, given in Table 2 for equilibrium configurations. Note also that $N_{s}=2\left(n_{\rho}+n_{\lambda}\right)+\left(l_{\rho}+l_{\lambda}\right)$ denotes the excitation in spherical orbitals such that $N_{s} \geqq N$, the excitation in deformed orbitals. As a consequence of choosing the $z$-axis as the axis of symmetry, $m_{p}=0$ $=m_{\lambda}$ in the intrinsic or body fixed coordinate system. Parity considerations restrict the sum over $l_{\rho}$ in (2.8) to even or odd only depending on whether $\tilde{N}$ $=\left(N_{\rho_{x}}+N_{\rho_{y}}+N_{\rho_{z}}\right)$ is even or odd. Consider an intrinsic state denoted by $\psi_{P}^{N}(\rho, \lambda)$ where $P$ specifies the permutation symmetry. We wish to project out from this intrinsic eigenstates of $L^{2}$ and $L_{3}$, where $\mathbf{L}=\mathbf{L}_{\rho}$ $+\mathbf{L}_{\lambda}$, and $L_{3}$ is its projection about the space-fixed 3 -axis. Denoting the eigenvalues of $L^{2}$ and $L_{3}$ by $l(l$ 
Table 2. The expansion coefficients $C_{n i}^{(\tilde{N})}$ in a spherical oscillator basic defined by (2.8) are shown at equilibrium. The expansion is truncated at $N_{s}(=2 n+l)=6$ to a good approximation.

\begin{tabular}{|c|c|c|c|c|c|c|}
\hline \multirow[t]{2}{*}{$l$} & \multirow[t]{2}{*}{$n$} & \multicolumn{2}{|l|}{$N=1$} & \multicolumn{3}{|l|}{$N=2$} \\
\hline & & $\psi_{000}$ & $\psi_{001}$ & $\psi_{000}$ & $\psi_{001}$ & $\psi_{002}$ \\
\hline \multirow[t]{4}{*}{0} & 0 & 0.9803 & - & 0.9517 & - & -0.2361 \\
\hline & 1 & 0.0012 & - & 0.0045 & - & -0.4831 \\
\hline & 2 & 0.0139 & - & 0.0328 & - & 0.1405 \\
\hline & 3 & -0.0009 & - & -0.0032 & - & -0.0663 \\
\hline \multirow[t]{3}{*}{1} & 0 & - & 0.9547 & - & 0.8913 & - \\
\hline & 1 & - & -0.1361 & - & -0.1944 & - \\
\hline & 2 & - & 0.0323 & - & 0.0716 & - \\
\hline \multirow[t]{3}{*}{2} & 0 & 0.1935 & - & 0.2922 & - & 0.609 \\
\hline & 1 & -0.0114 & - & -0.0256 & - & -0.3439 \\
\hline & 2 & 0.0040 & - & 0.0144 & - & 0.1296 \\
\hline \multirow[t]{2}{*}{3} & 0 & - & 0.2529 & - & 0.3671 & - \\
\hline & 1 & - & -0.0402 & - & -0.0890 & - \\
\hline \multirow[t]{2}{*}{4} & 0 & 0.0336 & - & 0.0788 & - & 0.3480 \\
\hline & 1 & -0.0032 & - & -0.0112 & - & -0.1535 \\
\hline 5 & 0 & - & 0.0545 & - & 0.1230 & - \\
\hline 6 & 0 & 0.0058 & - & 0.0120 & - & 0.1434 \\
\hline
\end{tabular}

$+1)$ and $m$, the projected state of good angular momentum is given by $[23,24]$

$\psi_{P l m}^{N}(\rho, \lambda)=\int \mathscr{D}_{m 0}^{l}(\Omega) R_{\lambda}(\Omega) \psi_{P}^{N}(\rho, \lambda) d \Omega$,

where $\mathscr{D}_{m 0}^{i}(\Omega)$ is the usual $\mathscr{D}$-matrix, and $R_{\lambda}, R_{\rho}$ are rotation operators as defined in [24]. After some algebra, this reduces to

$$
\begin{aligned}
& \Psi_{P l m}^{N}=R_{P l}^{N}\left(\frac{4 \pi}{2 l+1}\right) \sum_{l_{\rho} l_{\lambda n_{\rho} n_{\lambda}}} \mathscr{F}_{P}^{(N)}\left(l_{\rho} l_{\lambda} ; n_{\rho} n_{\lambda}\right) \\
& \cdot(2 l+1)^{\frac{1}{2}}(-1)^{l_{\rho}-l_{\lambda}}\left(\begin{array}{ccc}
l_{\rho} & l_{\lambda} & l \\
0 & 0 & 0
\end{array}\right)\left(\phi_{n_{\rho} l_{\rho}}(\rho) \otimes \phi_{n_{\lambda} l_{\lambda}}(\lambda)\right)_{l m},
\end{aligned}
$$

where $\otimes$ denotes a spherical tensor product. The relevant $\mathscr{F}$ 's used in this calculation are given by

$$
\begin{aligned}
& \mathscr{F}_{\rho}^{(1)}\left(l_{\rho} l_{\lambda} ; n_{\rho} n_{\lambda}\right)=C_{n_{\rho} l_{\rho}}^{(1)} C_{n_{\lambda} l_{\lambda}}^{(0)}, \\
& \mathscr{F}_{\lambda}^{(1)}\left(l_{\rho} l_{\lambda} ; n_{\rho} n_{\lambda}\right)=C_{n_{\rho} l_{\rho}}^{(0)} C_{n_{\lambda} l_{\lambda}}^{(1)}, \\
& \mathscr{F}_{\mathrm{sym}}^{(2)}\left(l_{\rho} l_{\lambda} ; n_{\rho} n_{\lambda}\right)=\frac{1}{\sqrt{2}}\left(C_{n_{\rho} l_{\rho}}^{(2)} C_{n_{\lambda} l_{\lambda}}^{(0)}+C_{n_{\rho} l_{\rho}}^{(0)} C_{n_{\lambda} l_{\lambda}}^{(2)}\right), \\
& \mathscr{F}_{\lambda}^{(2)}\left(l_{\rho} l_{\lambda} ; n_{\rho} n_{\lambda}\right)=\frac{1}{\sqrt{2}}\left(C_{n_{\rho} l_{\rho}}^{(2)} C_{n_{\lambda} l_{\lambda}}^{(0)}-C_{n_{\rho} l_{\rho}}^{(0)} C_{n_{\lambda} l_{\lambda}}^{(2)}\right), \\
& \mathscr{F}_{\beta}^{(2)}\left(l_{\rho} l_{\lambda} ; n_{\rho} n_{\lambda}\right)=C_{n_{\rho} l_{\rho}}^{(1)} C_{n_{\lambda} l_{\lambda}}^{(1)} .
\end{aligned}
$$

The normalization factor $R_{P l}^{N}$ is given by

$\left(R_{P l}^{N}\right)^{-2}=\frac{(4 \pi)^{2}}{(2 l+1)} \sum_{l_{\rho} l_{\lambda} n_{\rho} n_{\lambda}}\left[\mathscr{F}_{P}^{(N)}\left(l_{\rho} l_{\lambda} ; n_{\rho} n_{\lambda}\right)\right]^{2}\left(\begin{array}{ccc}l_{\rho} & l_{\lambda} & l \\ 0 & 0 & 0\end{array}\right)^{2}$

The projected states of good orbital angular momentum $\Psi_{P: m}^{N}(\rho, \lambda)$ are therefore completely determined through (2.10), with the numerical values of the expansion coefficients $C_{n l}^{(\tilde{N})}$ given in Table 2. It is now straight-forward to couple the states (2.10) of good $l$ with spin eigenstates of $S=\frac{1}{2}$ or $S=\frac{3}{2}$ to construct states of definite $J$. A typical such coupled state, for example, is denoted by $\left|70^{-}, N=1,(S, l) J M\right\rangle$, and for the nucleon is given by

$$
\begin{aligned}
& \left|70^{-}, N=1,\left(\frac{1}{2}, 1\right) J M\right\rangle \\
& =\sum_{m}(2 J+1)^{\frac{1}{2}}(-)^{M-\frac{1}{2}}\left(\begin{array}{ccc}
\frac{1}{2} & 1 & J \\
\mu & m & -M
\end{array}\right) \\
& \cdot \frac{1}{2}\left\{\psi_{\rho, 1 m}^{1}\left(\chi_{\mu}^{\rho} \phi^{\lambda}+\chi_{\mu}^{\lambda} \phi^{\rho}\right)+\psi_{\lambda, 1 m}^{1}\left(\chi_{\mu}^{\rho} \phi^{\rho}+\chi_{\mu}^{\lambda} \phi^{\lambda}\right)\right\} .
\end{aligned}
$$

The notation for the spin-isospin wave functions is given in full in [11].

It may be noted that because of the state dependence of the mean field, the state $156^{+}, N$ $=2,(1 / 2,0), 1 / 2 M\rangle$ is not orthogonal to $\mid 56^{+}, N$ $=0,(1 / 2,0) 1 / 2 M\rangle$. In diagonalizing the full Hamiltonian $H$, we used the orthogonal basis in which the state $\left|56^{+}, N=2,(1 / 2,0), 1 / 2 M\right\rangle$ is replaced by

$\left|\widetilde{56}^{+}, N=2,(1 / 2,0), 1 / 2 M\right\rangle=a \mid 56^{+}, N=2,(1 / 2,0), 1 / 2 M$

$+b\left|56^{+}, N=0,(1 / 2,0), 1 / 2 M\right\rangle$

where

$a=\frac{1}{\sqrt{1+\xi^{2}}}, \quad b=\frac{-\xi}{\sqrt{1+\xi^{2}}}$

and

$\xi=\left\langle 56^{+}, N=2,(1 / 2,0), 1 / 2 M\right|$

$\left.56^{+}, N=0,(1 / 2,0), 1 / 2 M\right\rangle$

is the overlap between the two non-orthogonal states. At equilibrium deformation $\xi=-0.318$. Note that $\left|\widetilde{56}^{+}, N=2,(1 / 2,0), 1 / 2 M\right\rangle$ is now orthogonal to $\left|56^{+}, N=0,(1 / 2,0), 1 / 2 M\right\rangle$.

\section{The Hamiltonian}

The complete Hamiltonian is expressed in the following form,

$H=H_{0}+V_{\mathrm{OGE}}+V_{\mathrm{OPE}}+V_{\mathrm{SO}}$.

The first term is the mean deformed Hamiltonian discussed in Sect. 2. The second term is obtained by 
a Breit-Fermi reduction of the OGE amplitude [25]. The third term is the contribution of the OPE potential between valence quarks. The last term is a one body spin-orbit potential arising from the confinement potential. In the following we shall discuss these terms one by one.

The OGE potential can be written as follows:

$$
\begin{aligned}
V_{\mathrm{OGE}}= & V_{C}^{G}+V_{\sigma}^{G}+V_{t}^{G}+V_{\mathrm{SO}}^{G} \\
& +\ldots \text { momentum dependent terms. }
\end{aligned}
$$

In the static approximation the momentum dependent terms are neglected. The detailed reduction of the OGE potential has been discussed in detail (see for example the review by Hey and Kelly [25]) and therefore we shall give only the final expressions using the symmetry properties. The first term in (3.2) is the spin independent central interaction given by

$V_{c}^{G}=-\sqrt{2} \frac{\alpha_{s}}{\rho}+\frac{\pi \alpha_{s}}{\sqrt{2} m^{2}} \delta^{3}(\rho)$

which consists of the attractive Coulomb interaction and a repulsive delta function interaction often referred to as the "Darwin term". The latter is normally ignored in the literature, but we retain it as its contribution is of the same order as the spin dependent terms. Hereafter $\alpha_{s}$ denotes the strength of the gluon coupling to quark.

The second term in (3.2) is the central spin-dependent interaction given by

$V_{a}^{G}=\frac{\sqrt{2} \pi \alpha_{s}}{3 m^{2}} \sigma_{1} \cdot \sigma_{2} \delta^{3}(\rho)$,

and the third term is the tensor interaction

$V_{t}^{G}=\frac{\alpha_{s}}{4 \sqrt{2} m^{2}} \frac{1}{\rho^{3}} S_{12} ; \quad S_{12}=3 \sigma_{1} \cdot \hat{\rho} \sigma_{2} \cdot \hat{\rho}-\sigma_{1} \cdot \sigma_{2}$.

The last term in (3.2) is the spin-orbit force,

$V_{\mathrm{SO}}^{G}=\frac{3 \alpha_{s}}{4 \sqrt{2} m^{2}} \frac{1}{\rho^{3}}$

$\left\{\left(\sigma_{1}+\sigma_{2}\right) \cdot \boldsymbol{\rho} \times \mathbf{p}_{\rho}-\frac{1}{3 \sqrt{3}}\left(\sigma_{1}-\sigma_{2}\right) \cdot\left(\boldsymbol{\rho} \times \mathbf{p}_{\lambda}\right)\right\}$.

The first part of the above potential is a two body spin-orbit force while the second term is a genuine three body spin-orbit force. In fact in deriving $V_{\text {SO }}^{G}$ one starts with a pure two body spin orbit force between any two valence quarks. The elimination of the centre of mass dependence leads to (3.6). For a detailed discussion see Gromes [13] and Reinders [26].
In the pseudoscalar coupling the one pion exchange potential $V_{\mathrm{OPE}}$ in the static approximation is given by (for point pions)

$$
V_{\mathrm{OPE}}\left(\mathrm{r}_{i j}\right)=-\frac{1}{(2 \pi)^{3}} \frac{\pi \alpha_{\pi}}{m^{2}} \tau_{i} \cdot \tau_{j} \int d^{3} q e^{i \mathbf{q} \cdot \mathbf{r}_{i j}} \frac{\boldsymbol{\sigma}_{i} \cdot \mathbf{q} \boldsymbol{\sigma}_{j} \cdot \mathbf{q}}{q^{2}+m_{\pi}^{2}}
$$

where $\alpha_{\pi}=\frac{g_{q q \pi}^{2}}{4 \pi}$ and $\mathbf{q}$ denotes the three momentum of the exchanged pion. The OPE potential between quarks is written in analogy with the OPE potential in $N-N$ scattering. We modify this by introducing a form factor at the $\pi-q-q$ vertex [27],

$$
F_{\pi}\left(q^{2}\right)=\frac{m_{\rho}^{2}}{m_{\rho}^{2}+q^{2}}
$$

to take into account the finite size of the pion. The pion radius is about $0.63 \mathrm{fm}$ if we choose $m_{\rho}$ $=770 \mathrm{MeV}$, the $\rho$-meson mass. $V_{\text {OPE }}$ can now be separated into a central spin-spin and tensor components,

$$
V_{\mathrm{OPE}}=V_{\sigma}^{\pi}+V_{t}^{\pi},
$$

where

$$
\begin{aligned}
& V_{\sigma}^{\pi}=-\frac{\alpha_{\pi}}{4 m^{2}}\left(\frac{m_{\rho}^{2}}{m_{\rho}^{2}-m_{\pi}^{2}}\right)^{2} \sigma_{1} \cdot \sigma_{2} \tau_{1} \cdot \tau_{2}\left[\frac{m_{\rho}^{3}}{2}\left(1-\frac{m_{\pi}^{2}}{m_{\rho}^{2}}\right)\right. \\
& \left.\cdot e^{-\sqrt{2} m_{\rho} \rho}-\frac{m_{\pi}^{2}}{\sqrt{2}}\left(\frac{e^{-\sqrt{2} m_{\pi} \rho}}{\rho}-\frac{e^{-\sqrt{2} m_{\rho} \rho}}{\rho}\right)\right]
\end{aligned}
$$

and

$$
\begin{aligned}
& V_{t}^{\pi}=\frac{\alpha_{\pi}}{4 m^{2}}\left(\frac{m_{\rho}^{2}}{m_{\rho}^{2}-m_{\pi}^{2}}\right)^{2} t_{1} \cdot \tau_{2} S_{12}\left[f\left(\sqrt{2} m_{\pi} \rho\right)\right. \\
& \left.-f\left(\sqrt{2} m_{\rho} \rho\right)-\frac{1}{2}\left(m_{\rho}^{2}-m_{\pi}^{2}\right)\left(m_{\rho}+\frac{1}{\sqrt{2} \rho}\right) e^{-\gamma 2 m_{\rho} \rho}\right]
\end{aligned}
$$

with

$f(\mu \rho)=\frac{\mu^{2} e^{-\mu \rho}}{2 \sqrt{2} \rho}\left(1+\frac{3}{\mu \rho}+\frac{3}{\mu^{2} \rho^{2}}\right)$.

In the event of $F_{\pi}=1$, the $V_{\text {OPE }}$ takes on a simple form given by

$$
\begin{aligned}
& V_{\rho}^{\pi}=-\frac{\alpha_{\pi}}{4 m^{2}} \tau_{1} \cdot \tau_{2} \sigma_{1} \cdot \sigma_{2}\left[\frac{4 \pi}{2 \sqrt{2}} \delta^{3}(\rho)-m_{\pi}^{2} \frac{e^{-\sqrt{2} m_{\pi} \rho}}{\sqrt{2} \rho}\right], \\
& V_{t}^{\pi}=\frac{\alpha_{\pi}}{4 m^{2}} \tau_{1} \cdot \tau_{2} S_{12} f\left(\sqrt{2} m_{\pi} \rho\right) .
\end{aligned}
$$

The effect of the form factor is to suppress the short ranged components of OPE more than the tail. It 
was shown in [12] that for a point pion, $\alpha_{\pi}=0.35$, with $\alpha_{s}=0.35$ yielded the $N-\Delta$ splitting. With the choice of the form factor (3.8), we need $\alpha_{\pi}$ close to unity for the same $\alpha_{s}=0.35$. Whereas the spectroscopic calculations may be performed with or without the pion form factor, the resulting spectra are better if the form factor is used. This is because the OPE tensor force is largely suppressed by the form factor, which is desirable, as will be discussed shortly. A value of $\alpha_{\pi}$ close to unity is also obtained by the pion decay-widths of the resonances [28] in the simple quark model. Note also that unlike the onegluon-exchange potential, $V_{\mathrm{OPE}}$ is flavour-dependent, and its matrix-elements for the $N$ - and $\Delta$-states are very different.

The last term in (3.1) is the one-body spin-orbit potential, put in phenomenologically to simulate the spin-orbit splitting that would arise from a relativistic treatment of the scalar confinement potential. In earlier papers $[2,26]$, this potential is referred to as the Thomas term, and is obtained by the appropriate nonrelativistic reduction of the Dirac equation. We estimate the term differently, by directly calculating the spectrum of a zero-mass current quark in the relativistic case, and choosing the strength of $V_{\text {so }}$ in (3.1) to reproduce the spin-orbit splitting. This approach is in the spirit of an earlier paper [29] which attempted to justify the constituent quark model. Consider first a zero mass quark moving in a spherical cavity of fixed radius $R$. By solving the Dirac equation, we know that the $p_{1 / 2}-p_{3 / 2}$ splitting is $0.61 / R$, and the $d_{3 / 2}-d_{5 / 2}$ splitting is $0.79 / R$. The rms single-particle radius of the quark is $0.73 R$ in its ground state. We now take a constituent quark of constituent mass $m$, moving nonrelativistically in an oscillator potential and a constant spin-orbit field:

$V_{i}=\frac{1}{2} m \omega_{0}^{2} r_{i}^{2}-\frac{C}{2} \mathbf{I}_{i} \cdot \boldsymbol{\sigma}_{i}$

where $C$ is a constant in a given shell. The single particle rms radius in the ground state is now $\left\langle r^{2}\right\rangle^{\frac{1}{2}}$ $=\sqrt{\frac{3}{2} \frac{\hbar}{m \omega_{0}}}$ and for our parameters $\hbar \omega_{0}=550 \mathrm{MeV}$, $m=330 \mathrm{MeV},\left\langle r^{2}\right\rangle^{\frac{1}{2}}=0.695 \mathrm{fm}$. For the relativistic case, the same rms radius would be obtained if the cavity radius is taken to be $R=0.95 \mathrm{fm}$. The constant $C$ for the $p$-shell is now chosen so as to reproduce the spin-orbit relativistic splitting for a bag of this size, i.e., $\frac{3}{2} C=\frac{0.61}{R}$, so $C=85 \mathrm{MeV}$ for $R$ $=0.95 \mathrm{fm}$ in the $p$-shell. Similarly, in the $d$-shell, $\frac{5}{2} C$ $=\frac{0.79}{R}$, so $C=65 \mathrm{MeV}$. Admittedly, this procedure is very rough, but gives us a guideline for choosing the strength of the one-body spin-orbit field that would approximately yield the relativistic splittings. If, instead of fitting the ground state size, we had chosen to fit the energy spacings between the shells in the two models, the radius $R$ would be almost half as large and the strength $C$ correspondingly larger. As a guideline then, we expect the strength $C$ to be in the range $80-160 \mathrm{MeV}$ for the $p$-shell, and between $60-120 \mathrm{MeV}$ in the $d$-shell. The one-body spin-orbit potential in (3.1) is thus taken as

$V_{\mathrm{SO}}=-\frac{C}{2} \sum_{i} \mathbf{1}_{i} \cdot \boldsymbol{\sigma}_{i}$

which goes over to

$V_{\mathrm{SO}}=-C \boldsymbol{\sigma}_{3} \cdot\left(\lambda \times \mathbf{p}_{\lambda}\right)$.

For our numerical work, we found good fits for

$$
\begin{aligned}
C & =100 \mathrm{MeV}(N=1 \text { states }), \\
& =50 \mathrm{MeV}(N=2 \text { states }),
\end{aligned}
$$

which are not in conflict with the rough estimates above.

Having specified the residual interaction completely, we are now in a position to diagonalize the interaction given by (3.1) in the space of deformed states. The procedure is a follows: First the Hamiltonian $\left(H_{0}+V_{C}^{G}+V_{\sigma}^{G}+V_{\sigma}^{\pi}\right)$, excluding the tensor and spin-orbit parts of the total Hamiltonian, is diagonalized in the space of intrinsic (unprojected) states. These intrinsic states, constructed from the space and spin-isospin-wave-functions of appropriate symmetry, are explicitly given in [11], (3.14)-(3.26). This takes care of the anharmonic components in the interaction which might have been left out from $H_{0}$. Since the mean field is state dependent, the intrinsic states corresponding to different $N$ but having the same spin, isospin and permutation symmetry are not mutually orthogonal. By using the prescription given in [11] we calculate the off-diagonal matrix element of $H_{0}$ between non-orthogonal states. The good angular momentum states are then projected out. With sufficiently well deformed states ${ }^{\star}$ projection onto states of good $l$ generates $[23,24]$ the characteristic rotational spectrum built on a particular band head. The energy of the band head is given by

\footnotetext{
* The proof of this statement follows from the fact that $\left\langle e^{-i \theta_{2} L_{2}}\right\rangle$, where $\theta_{2}$ is the angle of rotation perpendicular to the axis of symmetry for prolate deformations, should behave $[23,24]$ as $e^{-\theta_{2}^{2}\left\langle L^{2}\right\rangle / 4}, 0 \leqq \theta_{2} \leqq \frac{\pi}{2}$. This is indeed the case with deformed states under consideration
} 
$E(l=0)=E_{\text {intrinsic }}-\frac{\hbar^{2}\left\langle L^{2}\right\rangle}{2 \mathscr{I}}$

for even-parity prolate states and

$E(l=1)=E_{\text {intrinsic }}+\frac{\hbar^{2}}{2 \mathscr{I}}\left(2-\left\langle L^{2}\right\rangle\right)$

for odd-parity prolate states. The higher $l$ states are obtained by noting that the energy increases as $\frac{\hbar^{2}}{2 \mathscr{I}} l(l+1)$ in each band. The moment of inertia $\mathscr{I}$ and $\left\langle L^{2}\right\rangle$ are given at equilibrium in [11]:

$\mathscr{J}=\hbar\left[\frac{N_{z}+1}{\omega_{z}}+\frac{N_{y}+1}{\omega_{y}}\right] ; \quad\left\langle L^{2}\right\rangle=\left(\frac{\omega_{\rangle}}{\omega_{\zeta}}\right)^{2}-1$,

where $\omega_{\rangle}\left(\omega_{\langle}\right)$is the larger (smaller) of $\omega_{y}$ and $\omega_{z}$, when $\omega_{x}=\omega_{y}$.

The good $l$ states are coupled to spin $S$ of the three-quark system to generate states of good $J$ which are degenerate for a given $l$ and $S$ at this stage. The non-central interactions, namely the spinorbit and tensor, are diagonalised at this stage to provide the approximate splittings and mixing. Ideally the complete diagonalization should have been carried out in the space of intrinsic states. Our procedure to diagonalize the non-central interaction is only approximate but a good one as the spin-orbit and tensor couplings are rather small compared to the central interaction. We do not expect the deformation to change from the equilibrium value because of this.

\section{Results and Discussion}

The energy spectra of the nucleon and the delta obtained in our model are compared with the available experimental data in Figs. 1-3. In Table 3, the corresponding eigenstates are given in the basis of the orthogonalised projected states. The parameters of the model are given by

$\alpha_{s}=0.35, \quad \alpha_{\pi}=0.95, \quad \hbar \omega_{0}=550 \mathrm{MeV}$,

$m=330 \mathrm{MeV}, \quad C(N=1)=100 \mathrm{MeV}$,

$C(N=2)=50 \mathrm{MeV}$, and $E_{c}=-1,262 \mathrm{MeV}$.

Here $E_{c}$ is an overall constant which is added to the total Hamiltonian to fix the nucleon ground state at $940 \mathrm{MeV}$. The rationale for the choices of $C$, the strength of the one-body spin-orbit potential, and $\alpha_{\pi}$, the $q q \pi$-coupling parameter, have been discussed earlier. We now discuss the reasons for the choice of the other parameters in (4.1).

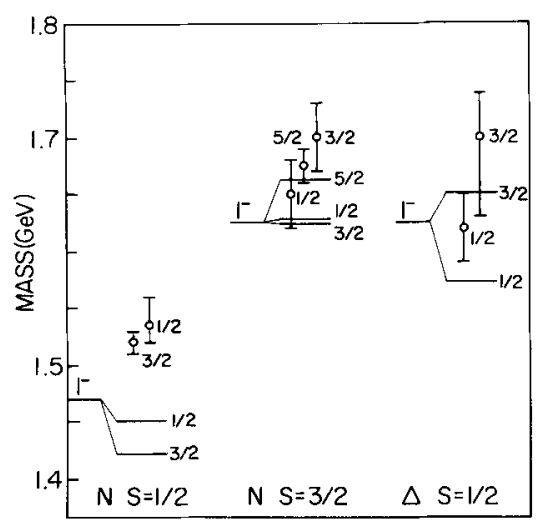

Fig. 1. The low-lying odd-parity states of the nucleon and delta. The experimental data are taken from [1] and are shown by open circles. The error bars indicate the uncertainty in the nomial masses. The position of the projected $l=1$ states is indicated in the figure for reference. The calculated positions of the good- $J$ states are shown by horizontal lines. The corresponding wavefunctions for these states in the deformed oscillator basis are given in Table 3 , (a) and (b)

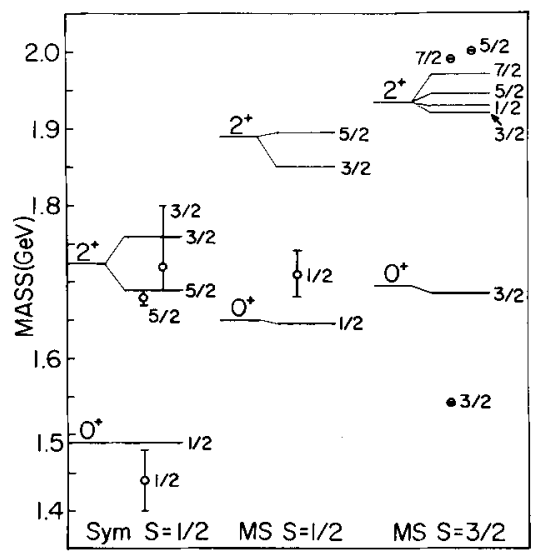

Fig. 2. The low-lying even-parity states of the nucleon. The legend is in the same style as Fig. 1. The open circles without error bars denote experimental states which are not well established. The first two members of the rotational band, $0^{+}$and $2^{+}$, which are projected from the deformed intrinsic states, are also indicated for reference. The structure of the wavefunctions of the states is given in Table 3,(c)

In $\mathrm{QCD}$, the quark-gluon running coupling constant is

$\alpha_{s}\left(q^{2}\right)=12 \pi /\left[\left(33-2 n_{f}\right) \ln \left(\frac{q^{2}}{\Lambda^{2}}\right)\right]$,

where $n_{f}$ is the number of flavours and $A$ is the scale parameter. The above expression should not be used for $q^{2} \approx \Lambda^{2}$. At $q^{2}=1 \mathrm{GeV}^{2}$, and for $\Lambda$ between 0.1 and $0.2 \mathrm{GeV}$, and $n_{f}=3, \alpha_{s}$ is in the range of 0.3 to 0.4. For our calculation, we take $\alpha_{s}$ to be a constant at 0.35 , noting that the energy scale in the baryonic spectra is in the range of $1 \mathrm{GeV}$. The con- 


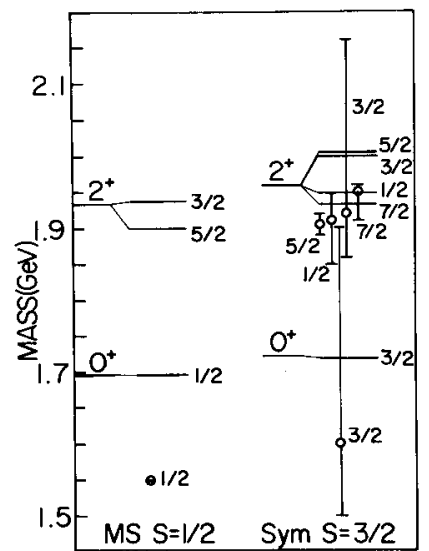

Fig. 3. The low-lying even-parity states of the delta. The legend is in the same style as in Fig. 2. The structure of the wavefunctions is given in Table 3, (d). Note, as in Fig. 2, that even-parity states whose dominant component is mixed symmetric (70) are mostly weakly seen, or not seen experimentally

stituent quark mass $m=0.33 \mathrm{GeV}$ is the standard value used to reproduce the ground state nucleon magnetic moment. Recently, it has been suggested [30] that the constituent quark mass may be viewed as the dynamically generated mass due to a nonperturbative infinite sum of gluonic radiative corrections on a quark-line. It is shown that by taking $q^{2}$ $=1 \mathrm{GeV}^{2}$, and $\alpha_{s}=0.4$, one obtains $m \approx 300 \mathrm{MeV}$ for zero-mass current quarks. Here, of course, pion effects are not included. It may be argued that pionic currents may contribute about half the observed nucleon magnetic moment [16] for a quark-core of radius $0.6-0.7 \mathrm{fm}$. Such a reduction in the quark contribution to the magnetic moment should presumably be accounted for by a corresponding increase in the constituent quark mass. We emphasize that our calculated spectrum is not sensitive to the choice of $m$. Except for the Coulomb term, the matrix-elements of the other residual interactions scale as $\alpha \sqrt{\omega_{0}^{3} / m}$, where $\alpha$ is $\alpha_{s}$ or $\alpha_{\pi}$. An increase in $m$ by a factor of 2 , for example, may be accommodated by increasing $\alpha$ by a corresponding factor of $\sqrt{2}$, or even less if $\omega_{0}$ is changed slightly. The Coulomb interaction, on the other hand, scales as $\alpha_{s} \sqrt{m \omega_{0}}$, but is rather weak compared to other matrix-elements. The net result of increasing $m$, even by a factor of two, should not alter the quality of fit too much. This was confirmed for the case of $N=1$ oddparity states, where the results actually improved slightly with the enhanced strength of the Coulomb term. Finally, the oscillator parameter $\hbar \omega_{0}$, which determines the moment of inertia $\mathscr{I}$ (3.21), was chosen to reproduce the spacings between the members of the rotational band. The results would not change much so long as it remained in the range 500-
$600 \mathrm{MeV}$. The deformations of the excited states were determined through (2.4), and these, in turn, determined $\left\langle L^{2}\right\rangle$ for a given intrinsic state, irrespective of $\omega_{0}(3.21)$.

Consider now the $N=1$ odd parity spectrum of Fig. 1. The $N=3$ configuration mixing with $N=1$, which is only about $4 \%$ in the deformed basis, has been omitted. For a discussion of the $N=3$ lowlying states, the reader should see [12]. On the whole, we find that the calculated $N=1$ states are about 50 to $70 \mathrm{MeV}$ lower than the experimental values. This is partially due to the inclusion of the Darwin term (3.3). Its exclusion would improve the $N=1$ spectrum, but we retained it for consistency, and also because it helped in the $N=2$ states. Although the overall agreement is reasonable, there is some anomaly in the ordering of the $l=1, S=3 / 2$ states. We obtain a degenerate $1 / 2^{-}$and $3 / 2^{-}$, with $5 / 2^{-}$about $40 \mathrm{MeV}$ higher. The data, however, indicates $3 / 2^{-}$higher than the $5 / 2^{-}$and $1 / 2^{-}$states. This is partially due to the pionic tensor component, which, as a result of flavour dependence, has a sign opposite to the OGE tensor force in the nucleon. Hence the $3 / 2^{-}$is brought lower than the $5 / 2^{-}$in our model. If we had used a point pion, the result would be worse.

Thus the key to the solution of the spin-orbit puzzle may be the following: (1) The reduction in the value of $\alpha_{s}$ by a factor of three made possible by the pionic component of the spin-spin force contributing to little more than half of $N-\Delta$ splitting in the ground state. (2) A further suppression by about $30 \%$ due to the deformation of the $N=1$ states. These two factors combine to bring down the spinorbit contribution from OGE within manageable limits. The introduction of the one-body spin-orbit force $V_{\text {so }}$ not only cancels the remaining $V_{\text {so }}^{G}$ from the gluon exchange but also restores the correct ordering of the states in $l=1, S=1 / 2$ as well as improving the splitting between the two delta $l=1, S$ $=1 / 2$ states. It should be noted that while the spinorbit forces from OGE and the confining potential cancel in the nucleon states, they add up in the delta states. The choice of the constant $C$ ensures the right amount of splitting in the $l=1$ states.

The mixing between the $S=1 / 2$ and $S=3 / 2$ states is shown in Table 3. We find that the $J=3 / 2$ states are strongly mixed while the $J=1 / 2$ states are almost decoupled. This is just the opposite of what is found in the model of Isgur and Karl [2]. This is due to the fact that in a pure tensor coupling with no spin-orbit interaction, the $J=1 / 2$ states are more strongly mixed than the $J=3 / 2$ states. The inclusion of the spin-orbit forces reverses the situation. The off diagonal couplings of one-body $V_{\text {so }}$ and the OGE 
Table 3. The configuration mixings of various baryon excited states and the corresponding energies are given. These are obtained after diagonalizing the full Hamiltonian given in Sect. 3 at equilibrium deformed values. The experimental data are taken from [1]. The masses given in brackets correspond to the resonances which have not been identified very well. The states are denoted by $|(), N,(S, l) J\rangle$, where the bracket here stands for the $S U(6)$ multiplet, like $70^{-}$, etc.

\begin{tabular}{|c|c|c|c|c|}
\hline & & & \multicolumn{2}{|c|}{ Mass $(\mathrm{MeV})$} \\
\hline & & & Theory & Expt. \\
\hline \multicolumn{5}{|c|}{ (a) Odd parity nucleon states: configuration } \\
\hline $\begin{array}{l}\left|70^{-}, N=1,(1 / 2,1), 1 / 2\right\rangle \\
\left|70^{-}, N=1,(3 / 2,1), 1 / 2\right\rangle\end{array}$ & $\begin{array}{l}0.999 \\
0.014\end{array}$ & $\begin{array}{r}-0.014 \\
0.999\end{array}$ & $\begin{array}{l}1,451 \\
1,628\end{array}$ & $\begin{array}{l}1,520-1,560 \\
1,620-1,680\end{array}$ \\
\hline $\begin{array}{l}\left|70^{-}, N=1,(1 / 2,1), 3 / 2\right\rangle \\
\left|70^{-}, N=1,(3 / 2,1), 3 / 2\right\rangle\end{array}$ & $\mid \begin{array}{r}0.845 \\
-0.534\end{array}$ & $\begin{array}{l}0.534 \\
0.845\end{array} \mid$ & $\begin{array}{l}1,422 \\
1,624\end{array}$ & $\begin{array}{l}1,510-1,530 \\
1,670-1,730\end{array}$ \\
\hline$\left|70^{-}, N=1,(3 / 2,1), 5 / 2\right\rangle$ & & 1.0 & 1,663 & $1,660-1,690$ \\
\hline
\end{tabular}

(b) Odd parity delta states:

$\left|70^{-}, N=1,(1 / 2,1), 1 / 2\right\rangle$

$\left|70^{-}, N=1,(1 / 2,1), 3 / 2\right\rangle$

1.0

1,572

$1,600-1,650$

1.0

1,651

$1,630-1,740$

(c) Even parity nucleon states:

$\left|56^{-}, N=0,(1 / 2,0), 1 / 2\right\rangle$
$\left|\widetilde{56}^{+}, N=2,(1 / 2,0), 1 / 2\right\rangle$
$\left|70^{+}, N=2,(1 / 2,0), 1 / 2\right\rangle$
$\left|70^{+}, N=2,(3 / 2,2), 1 / 2\right\rangle$
$\left|70^{+}, N=2,(3 / 2,0), 3 / 2\right\rangle$
$\left|56^{+}, N=2,(1 / 2,2), 3 / 2\right\rangle$
$\left|70^{+}, N=2,(1 / 2,2), 3 / 2\right\rangle$
$\left|70^{+}, N=2,(3 / 2,2), 3 / 2\right\rangle$
$\left|56^{+}, N=2,(1 / 2,2), 5 / 2\right\rangle$
$\left|70^{+}, N=2,(1 / 2,2), 5 / 2\right\rangle$
$\left|70^{+}, N=2,(3 / 2,2), 5 / 2\right\rangle$
$\left|70^{+}, N=2,(3 / 2,2), 7 / 2\right\rangle$

\begin{tabular}{|c|c|c|}
\hline 0.996 & 0.061 & -0.067 \\
\hline-0.091 & 0.778 & -0.620 \\
\hline 0.017 & 0.624 & 0.768 \\
\hline-0.020 & 0.051 & 0.143 \\
\hline 0.950 & -0.107 & 0.265 \\
\hline 0.248 & 0.794 & -0.554 \\
\hline-0.100 & 0.543 & 0.750 \\
\hline-0.162 & 0.254 & 0.242 \\
\hline 0.753 & -0.610 & -0.248 \\
\hline 0.609 & 0.498 & 0.618 \\
\hline-0.253 & -0.615 & 0.746 \\
\hline
\end{tabular}

0.026
0.048
-0.143
0.988
0.126
-0.029
-0.364
0.922

940

1,487

1,646

1,928

$1,400-1,480$

$1,680-1,740$

0.554

0.750

0.242

1,683

1,760

1,849

1,919

$(1,540)$

$1,690-1,800$

$-$

1,692

1,894

1,943

$1,670-1,690$

$-$

$(2,000)$

1.0

1,950

$(1,990)$

(d) Even parity delta states:

$\left|70^{+}, N=2,(1 / 2,0), 1 / 2\right\rangle$

$\left|56^{+}, N=2,(3 / 2,2), 1 / 2\right\rangle$

0.999

0.028

$\left|56^{+}, N=0,(3 / 2,0), 3 / 2\right\rangle$

$\left|\tilde{56}^{+}, N=2,(3 / 2,0), 3 / 2\right\rangle$

$\left|70^{+}, N=2,(1 / 2,2), 3 / 2\right\rangle$

$\left|56^{+}, N=2,(3 / 2,2), 3 / 2\right\rangle$

$\left|70^{+}, N=2,(1 / 2,2), 5 / 2\right\rangle$

$\left|56^{+}, N=2,(3 / 2,2), 5 / 2\right\rangle$

$\left|56^{+}, N=2,(3 / 2,1), 7 / 2\right\rangle$

-0.028
0.999
0.179
0.980
-0.088
0.024
-0.422
0.905

1.0

$\begin{array}{ll}1,696 & (1,550) \\ 1,948 & 1,850-1,950 \\ 1,231 & 1,230-1,234 \\ 1,719 & 1,500-1,900 \\ 1,938 & - \\ 2,000 & 1,860-2,160 \\ 1,899 & - \\ 2,005 & 1,890-1,920 \\ 1,934 & 1,910-1,960\end{array}$

spin-orbit add constructively and are larger in $J=3 / 2$. Our result therefore is somewhat similar to Gromes [13] even though this comparison cannot be carried too far as we are dealing with deformed states.

The results for $N=2$ even-parity nucleon and delta states below $2 \mathrm{GeV}$ are presented in Figs. 2 and 3 respectively and the configuration mixings are given in Table 3. The agreement in general is good. We believe this is the first calculation which takes into account the spin-orbit contributions in the $N$ $=2$ even-parity states. Because of the larger deformation the spin-orbit force from OGE is suppressed even more than in $N=1$. The tensor forces from both OPE and OGE are small wherever they contribute and of the order of 20 to $30 \mathrm{MeV}$ only. The even-parity nucleon states around $1.7 \mathrm{GeV}$ and the delta states around $1.9 \mathrm{GeV}$ show a remarkable agreement with the observed value. As in our earlier calculation [12], the splitting between Roper $(1.44 \mathrm{GeV}) 1 / 2^{+}$and the $1 / 2^{+}(1.7 \mathrm{GeV})$ remains an enigma as this splitting is not affected by the introduction of the non-central components.

The configuration mixing, given in Table 3 , indicates a strong mixing between $S=1 / 2$ symmetric and mixed symmetry states in the nucleon spectrum. As explained earlier [10], such a strong mixing is 
necessary to explain the radiative decay amplitude of $N(1.44) 1 / 2^{+} \rightarrow N(0.94) 1 / 2^{+}+\gamma$ and the nearly vanishing amplitude for $N(1.71) 1 / 2^{+} \rightarrow N(0.94) 1 / 2^{+}$ $+\gamma$. A complete calculation of the radiative decay amplitudes and the mesonic decay widths of all the excited states is underway.

In conclusion, in this paper we attempt to resolve the spin-orbit problem, both for the odd- and even-parity low lying excited states, in a model which assumes the excited baryon to be deformed. The pion coupling to the quarks plays a very important role. The fits obtained for the $N=1$ odd parity states are comparable to those of Myhrer and Wroldsen [5] and Gromes [13]. The importance of deformation cannot be overemphasized for the $N=2$ even parity states - the lowering of the Roper resonance as well as the substatial suppression of the spin-orbit interaction take place through the deformation of the mean field. We should also emphasize that our treatment of the one-body spin-orbit term, $V_{\text {so }}$ in (3.1), is quite different from the procedure of replacing it by the Thomas term. Our treatment of the problem attempts to simulate the spectrum of a zero-mass current quark obeying the Dirac equation, and gives up the $1 / m$-expansion so far as the onebody field is concerned.

Acknowledgements. We are grateful to Yuki Nogami for discussions, Michiaki Nishimura for some valuable assistance in the numerical work, and Donald Sprung for a careful reading of the manuscript. This research was supported by a grant from the Natural Sciences and Engineering Research Council of Canada.

\section{Appendix}

In this appendix we give the spin-orbit and tensor matrix elements between the baryonic states defined by (2.9)-(2.13). They are of the general form

$$
\left\langle S^{\prime} l^{\prime}, J I\left|\mathcal{O}_{s}\right| S l ; J I\right\rangle,
$$

where $\mathcal{O}_{s}$ is any of the scalar operators in the OPE and OGE interactions discussed in Sect. 3. It acts on the quark spins $\mathbf{S}_{i}$ and isospins $\mathbf{t}_{i}$ and on the coordinates $\rho, \lambda$ in the coupled scheme, where

$$
\mathbf{S}=\mathbf{S}_{1}+\mathbf{S}_{2}+\mathbf{S}_{3}=\mathbf{S}_{12}+\mathbf{S}_{3} ; \quad \mathbf{I}=\mathbf{t}_{1}+\mathbf{t}_{2}+\mathbf{t}_{3}
$$

are the total intrinsic spin and isospin, respectively $(S=1 / 2,3 / 2$ and $I=1 / 2,3 / 2)$. The vector sum of the relative angular momenta in the $\rho$ - and $\lambda$-coordinates is $\mathbf{L}$ and the total spin of the baryon is $\mathbf{J}$ :

$\mathbf{L}=\mathbf{L}_{\rho}+\mathbf{L}_{\lambda} ; \quad \mathbf{J}=\mathbf{S}+\mathbf{L}$.

Since $\mathcal{O}_{s}$ is a scalar under rotations in normal space and isospin spaces, it will not change $J, I$ and their third components $M_{J}, I_{3}$. (The latter will be therefore omitted in our notation.)

We use the formalism given in Chap. VII of Edmonds [21] to decompose the matrix elements (1) into products of a spin/angular and a radial matrix element. The former is expressed in terms of $3 j, 6 j$ and $9 j$ symbols (in the notation of [31] using the Condon and Shortley phase convention); the latter are given in terms of the radial parts $R_{n_{\lambda} l_{\lambda}}(\lambda)$, $R_{n_{\rho} l_{\rho}}(\rho)$ of the spherical harmonic oscillator functions

$\phi_{n l m}(\mathbf{r})=R_{n l}(r) Y_{l m}\left(\Omega_{r}\right) \quad(\mathbf{r}=\boldsymbol{\rho}, \lambda)$

normalised as

$\int_{0}^{\infty} r^{2} R_{n l}^{2}(r) d r=1$

Since we work with deformed projected states which are expanded in the basis $\phi_{n i m}$ according to (2.10), all matrix elements (1) will be of the form

$$
\begin{aligned}
\left\langle S^{\prime} l^{\prime} ; J I\left|\mathcal{O}_{s}\right| S l ; J I\right\rangle \\
=\sum_{\substack{n_{\rho} n_{\lambda} l_{\rho} l_{2} l_{2} \\
n_{\rho} n^{\prime} l_{\rho}^{\prime} l_{\lambda}}}\left\langle n_{\rho}^{\prime} n_{\lambda}^{\prime} ; S^{\prime},\left(l_{\rho}^{\prime} l_{\lambda}^{\prime}\right) l^{\prime} ; J I\left|\mathcal{O}_{s}\right| n_{\rho} n_{\lambda} ; S,\left(l_{l} l_{\lambda}\right) l ; J I\right\rangle \\
\quad \cdot \mathscr{F}_{P}^{(N)}\left(l_{\rho} l_{\lambda} ; n_{\rho} n_{\lambda}\right) \mathscr{F}_{P}^{*}{ }^{*\left(N^{\prime}\right)}\left(l_{\rho}^{\prime} l_{\lambda}^{\prime} ; n_{\rho}^{\prime} n_{\lambda}^{\prime}\right) .
\end{aligned}
$$

In the following we shall for simplicity abbreviate the $n$ and $l$ quantum numbers by $\alpha$ and $\alpha^{\prime}$ :

$\alpha=\left(n_{\rho} n_{\lambda} l_{\rho} l_{\lambda}\right) ; \quad \alpha^{\prime}=\left(n_{\rho}^{\prime} n_{\lambda}^{\prime} l_{\rho}^{\prime} l_{\lambda}^{\prime}\right)$.

We also shall make use of the short-hand notation

$\hat{l}=\sqrt{2 l+1}, \quad \hat{S}=\sqrt{2 S+1}$, etc.

Where necessary, we shall also indicate the total spins $S_{12}, S_{12}^{\prime}$ (singlet or triplet) to which the quarks 1 and 2 are coupled, see (2).

a) 2-Body Spin-Orbit Force from $O G E$

$$
\begin{aligned}
\left\langle\alpha^{\prime} ; S^{\prime} l^{\prime} ; J I\left|\frac{1}{\rho^{3}}\left(\sigma_{1}+\sigma_{2}\right) \cdot 1_{\rho}\right| \alpha, S l ; J I\right\rangle & (-1)^{S+l^{\prime}+J}\left\{\begin{array}{lll}
J & l^{\prime} & S^{\prime} \\
1 & S & l
\end{array}\right\} \\
& \cdot\left\langle S^{\prime}|| \sigma_{1}+\sigma_{2}|| S\right\rangle\left\langle\alpha^{\prime}, l^{\prime}|| \frac{1}{\rho^{3}} I_{\rho}|| \alpha, l\right\rangle \\
= & \delta_{l_{\rho} l_{\rho}^{\prime}} \delta_{l_{\lambda} l_{\lambda}} \delta_{n_{\lambda} n_{\lambda}^{\prime}} \delta_{S_{12,1}} \delta_{S_{12}, 1}(-1)^{J+3 / 2+l^{\prime}} \\
& \cdot 2 \sqrt{6} \sqrt{l_{\rho}\left(l_{\rho}+1\right)} l_{\rho} \hat{S} \hat{S}^{\prime}\left\{\begin{array}{lll}
J & l^{\prime} & S^{\prime} \\
1 & S & l
\end{array}\right\}\left\{\begin{array}{lll}
1 & S^{\prime} & 1 / 2 \\
S & 1 & 1
\end{array}\right\} \\
& \cdot(-1)^{l_{\rho}+l_{\lambda}-l+1} \hat{M}\left\{\begin{array}{lll}
l_{\rho} & l^{\prime} & l_{\lambda} \\
l & l_{\rho} & 1
\end{array}\right\}\left\langle l_{\rho} n_{\rho}^{\prime}\left|\frac{1}{\rho_{3}}\right| l_{\rho} n_{\rho}\right\rangle .
\end{aligned}
$$

This force acts only in (12)-triplet states. 
b) 3-Body Spin-Orbit Force From OGE

$$
\begin{aligned}
&\left\langle\alpha^{\prime} S^{\prime} l^{\prime} ; J I\left|\frac{1}{\rho^{3}}\left(\boldsymbol{\sigma}_{1}-\boldsymbol{\sigma}_{2}\right) \cdot\left(\boldsymbol{\rho} \times \mathbf{p}_{\lambda}\right)\right| \alpha, S l ; J I\right\rangle \\
&=(-1)^{J+3 / 2+l_{\rho}+l_{\lambda}^{\prime}+l^{\prime}\left[1-(-1)^{S_{12}+S_{12}^{\prime}}\right]} \\
& \cdot m \omega_{0} 6 \hat{S} \hat{S^{\prime}} \hat{S}_{12} \hat{S}_{12}^{\prime} \hat{l}^{\hat{l}} \cdot \hat{l}_{\rho} \hat{l}_{\rho} \hat{l}_{\lambda} \hat{l}_{\lambda}\left(\begin{array}{ccc}
l_{\rho} & l_{\rho}^{\prime} & 1 \\
0 & 0 & 0
\end{array}\right)\left(\begin{array}{ccc}
l_{\lambda} & l_{\lambda}^{\prime} & 1 \\
0 & 0 & 0
\end{array}\right) \\
& \cdot\left\{\begin{array}{lll}
J & l^{\prime} & S^{\prime} \\
1 & S & l
\end{array}\right\}\left\{\begin{array}{ccc}
S_{12}^{\prime} & S^{\prime} & 1 / 2 \\
S & S_{12} & 1
\end{array}\right\}\left\{\begin{array}{ccc}
S_{12} & S_{12}^{\prime} & 1 \\
1 / 2 & 1 / 2 & 1 / 2
\end{array}\right\} \\
& \cdot\left\{\begin{array}{lll}
l_{\rho}^{\prime} & l_{\rho} & 1 \\
l_{\lambda}^{\prime} & l_{\lambda} & 1 \\
l^{\prime} & l & 1
\end{array}\right\}\left(2 n_{\lambda}^{\prime}+l_{\lambda}^{\prime}-2 n_{\lambda}-l_{\lambda}\right)\left\langle n_{\lambda}^{\prime} l_{\lambda}^{\prime}|\lambda| n_{\lambda} l_{\lambda}\right\rangle \\
& \cdot\left\langle n_{\rho}^{\prime} l_{\rho}^{\prime}\left|\frac{1}{\rho^{2}}\right| n_{\rho} l_{\rho}\right\rangle .
\end{aligned}
$$

This force connects only (12)- triplet to singlet states and vice versa. To express $\boldsymbol{\rho} \times \mathbf{p}_{\lambda}$ as a tensor operator of rank 1, we have made use of (5.1.8) of Edmonds [31] which, however, has to be corrected for a wrong sign.

c) 1-Body Spin-Orbit Force

$$
\begin{aligned}
& \left\langle\alpha^{\prime}, S^{\prime} l^{\prime} ; J I\left|\sigma_{3} \cdot \mathbf{l}_{\lambda}\right| \alpha, S l ; J I\right\rangle \\
& =\delta_{l_{\rho} l_{\rho}^{\prime}} \delta_{l_{\lambda} l_{\lambda}} \delta_{n_{\rho} n_{\rho}^{\prime}} \delta_{n_{\lambda} n_{\lambda}^{\prime}} \delta_{S_{12}, S_{12}^{\prime}}(-1)^{J+3 / 2+S_{12}+S+S^{\prime}+l^{\prime}} \\
& \cdot \sqrt{6} \sqrt{l_{\lambda}\left(l_{\lambda}+1\right)} \hat{l_{\lambda}} \hat{S} \hat{S^{\prime}}\left\{\begin{array}{lll}
J & l^{\prime} & S^{\prime} \\
1 & S & l
\end{array}\right\}\left\{\begin{array}{ccc}
1 / 2 & S^{\prime} & S_{12} \\
S & 1 / 2 & 1
\end{array}\right\} \\
& \cdot(-1)^{l_{\rho}+l_{\lambda}+l^{\prime}+1} \hat{l} \hat{l}\left\{\begin{array}{lll}
l_{\lambda} & l^{\prime} & l_{\rho} \\
l & l_{\lambda} & 1
\end{array}\right\} \text {. }
\end{aligned}
$$

Note that all three spin-orbit forces give proportional $J$-splittings, since their matrix elements depend on $J$ only through one and the same $6 j$-symbol.

\section{d) Tensor Force from $O G E$}

The tensor force, as is well known, acts only in (12)triplet states:

$$
\begin{aligned}
\left\langle\alpha^{\prime}, S^{\prime} l^{\prime} ; J I\left|\frac{1}{\rho^{3}}\left(\sigma_{1} \cdot \rho \sigma_{2} \cdot \rho-\frac{1}{3} \sigma_{1} \cdot \sigma_{2}\right)\right| \alpha, S l ; J I\right\rangle \\
=\delta_{l_{\lambda} l_{\lambda}^{\prime} \delta_{n_{\lambda} n_{\lambda}^{\prime}} \delta_{S_{12,1}^{\prime}} \delta_{S_{12,1}}(-1)^{J+\frac{1}{2}}} \\
\cdot 2 \sqrt{\frac{10}{3}} \hat{S} \hat{S}^{\prime} \hat{l}_{\rho} \hat{l}_{\rho}\left(\begin{array}{lll}
l_{\rho} & l_{\rho}^{\prime} & 2 \\
0 & 0 & 0
\end{array}\right)\left\{\begin{array}{lll}
J & l^{\prime} & S^{\prime} \\
2 & S & l
\end{array}\right\}\left\{\begin{array}{ccc}
2 & S & S^{\prime} \\
1 / 2 & 1 & 1
\end{array}\right\} \\
\cdot(-1)^{l_{\lambda}+l+l^{\prime}} \hat{l}\left\{\begin{array}{lll}
l_{\rho}^{\prime} & l^{\prime} & l_{\lambda} \\
l & l_{\rho} & 2
\end{array}\right\}\left\langle l_{\rho}^{\prime} n_{\rho}^{\prime}\left|\frac{1}{\rho^{3}}\right| l_{\rho} n_{\rho}\right\rangle .
\end{aligned}
$$

Note the different $J$ dependence from that of the spin-orbit force matrix elements.

\section{e) Tensor Force from $O P E$}

The matrix elements of the OPE tensor force can be obtained also from (12), replacing the radial matrix element of $\rho^{-3}$ by that of the more involved Yukawa-type function appearing in the square brackets of (3.11), and multiplying with the isospin matrix elements.

$$
\begin{aligned}
& \left\langle\phi^{\rho}\left|\tau_{1} \cdot \tau_{2}\right| \phi^{\rho}\right\rangle=-3 ; \\
& \left\langle\phi^{\hat{\lambda}}\left|\tau_{1} \cdot \tau_{2}\right| \phi^{\lambda}\right\rangle=\left\langle\phi^{s}\left|\tau_{1} \cdot \tau_{2}\right| \phi^{s}\right\rangle=1 .
\end{aligned}
$$

The Yukawa-type radial matrix elements are, although they can in principle be given analytically in terms of error functions, most conventiently integrated numerically.

Note that the phases of the above off-diagonal matrix elements depend on the order of coupling spin $\mathbf{S}$ and angular momentum $\mathbf{L}$ to the total spin $\mathbf{J}$. Our choice is the same as in Edmonds [31] and also as the one used by Gromes [13]. (All the particular spin-orbit and tensor matrix elements given by Gromes [13] for the spherical $N=1$ states agree with our above formulae.)

\section{References}

1. Review of Particle Properties: Rev. Mod. Phys. 56, No. 2 (1984)

2. N. Isgur, G. Karl: Phys. Rev. D18, 4187 (1978); D19, 2653 (1979); N. Isgur: In The new aspects of subnuclear physics, Erice, (1978), ed. A. Zichichi, p. 107. New York: Plenum 1980

3. C.P. Forsyth, R.E. Cutkosky: Z. Phys. C - Particles and Fields 18, 219 (1983)

4. T.A. DeGrand, R.L. Jaffe: Ann. Phys. (N.Y.) 100, 425 (1976); T.A. DeGrand: In Proc. of the IVth International Conference on Baryon Resonances, Toronto, (1980), ed. N. Isgur. Toronto: University of Toronto Press

5. F. Myhrer, J. Wroldsen: Z. Phys. C - Particles and Fields 25, 281 (1984)

6. H.R. Fiebig, B. Schwesinger: Nucl. Phys. A393, 349 (1983)

7. J. Carlson, J.B. Kogut, V.R. Pandharipande: Phys. Rev. D27, 233 (1983); D 28, 2807 (1983)

8. R. Koniuk, N. Isgur: Phys. Rev. D21, 1868 (1980)

9. B. Silvestre-Brac, C. Gignoux: I.S.N. Grenoble preprint (1984), to appear in Phys. Rev. D

10. R.K. Bhaduri, B. Jennings, J.C. Waddington: Phys. Rev. D29, 2051 (1984)

11. M.V.N. Murthy, M. Dey, J. Dey, R.K. Bhaduri: Phys. Rev. D30, 152 (1984)

12. M.V.N. Murthy, R.K. Bhaduri: Phys. Rev. Lett. 54, 745 (1985)

13. D. Gromes: Z. Phys. C - Particles and Fields 18, 249 (1983)

14. G.E. Brown, M. Rho, V. Vento: Phys. Lett. 84B, 383 (1979)

15. G.A. Miller, A.W. Thomas, S. Theberg: Phys. Lett. 91 B, 192 (1980); Phys. Rev. D22, 2823 (1980); A.W. Thomas: Adv. Nucl. Phys. 13, 1 (1983)

16. W. Weise: Quarks, chiral symmetry and dynamics of nuclear constituents. TPR-84-8 (University of Regensburg preprint). International Review of Nucl. Phys., Vol. I (1984), Singapore: World Scientific (to be published)

17. Y. Nogami, N. Ohtsuka: Phys. Rev. D26, 261 (1982) 
18. J. Navarro, V. Vento: Lett. 140B, 6 (1984)

19. T. Hastuda: Contribution B11 in the Tenth International Conference on Particles and Nuclei, Heidelberg, 1984

20. K. Brauer, A. Faessler, F. Fernandez, K. Shimizu: Tubingen University preprint (1984)

21. S.A. Chin: Phys. Lett. 109B, 161 (1982); Nucl. Phys. A382, 355 (1982); Y. Nogami, A. Suzuki: Prog. Theor. Phys. 69, $1184(1983)$

22. A. Bohr, B.R. Mottelson: Nuclear structure. Vol. II, p. 77. Reading, Mass.: Benjamin 1975

23. R.E. Peierls, J. Yoccoz: Proc. Phys. Soc. London 70, 381 (1957)
24. M.A. Preston, R.K. Bhaduri: Structure of the nucleus, p. 475477. Reading, Mass.: Wesley Addison 1975

25. A.J.G. Hey, R.L. Kelly: Phys. Rep. 96, 71 (1983)

26. L.J. Reinders: Baryon 1980, Ed. N. Isgur, p. 203. Toronto: University of Toronto 1980

27. D.C. Cheng, G.K. O'Neill: Elementary particle physics, p. 107. Reading, Mass.: Addison-Wesley 1979

28. M.A. Preston, A.K. Dutta: Phys. Rev. D 27, 2780 (1983)

29. R.K. Bhaduri, M. Brack: Phys. Rev. D25, 1443 (1982)

30. V. Elias, M.D. Scadron: Phys. Rev. D30, 647 (1984)

31. A.R. Edmonds: Angular momentum in quantum mechanics. Princeton: Princeton University Press 1957 